Journal of Patient-Centered

Volume 4

Issue 4 -- Health Disparities and Inequities: Part

Article 10 I

$11-6-2017$

\title{
Sensitivity of Current Methods for Diagnosing and Documenting Metabolic Syndrome Within a Large Community-Based Health Care System
}

Tiffany A. Mullen Danielle M. Greer Jennifer T. Fink

Renee E. Walker

Follow this and additional works at: https://aah.org/jpcrr

Part of the Cardiovascular Diseases Commons, Community Health and Preventive Medicine Commons, Diagnosis Commons, Endocrinology, Diabetes, and Metabolism Commons, and the Primary Care Commons

\section{Recommended Citation}

Mullen TA, Greer DM, Fink JT, Walker RE. Sensitivity of current methods for diagnosing and documenting metabolic syndrome within a large community-based health care system. J Patient Cent Res Rev. 2017;4:256-7.

Published quarterly by Midwest-based health system Advocate Aurora Health and indexed in PubMed Central, the Journal of Patient-Centered Research and Reviews (JPCRR) is an open access, peer-reviewed medical journal focused on disseminating scholarly works devoted to improving patient-centered care practices, health outcomes, and the patient experience. 


\section{Conference Proceedings: Aurora Scientific Day 2017}

The following abstracts were presented at the 43rd Annual Aurora Scientific Day research symposium, held May 24, 2017, in Milwaukee, Wisconsin. Aurora Scientific Day hosts a forum for original research conducted by faculty, fellows, residents, and other allied health professionals affiliated with Aurora Health Care, an integrated health system with hospital and clinic locations in eastern Wisconsin and northern Illinois.

FIRST PLACE POSTER

Assessment of Chronic Disease to Determine
Appropriateness of Implantable Cardioverter-
Defibrillator Therapy

Bilal M. Omery, Maharaj Singh, Randy S. Turkel, Robyn Shearer, Arshad Jahangir, M. Eyman Mortada, Jasbir S. Sra, Indrajit Choudhuri

Aurora Cardiovascular Services; Sheikh Khalifa bin Hamad Al Thani Center for Integrative Research on Cardiovascular Aging, Aurora Research Institute

Background: Implantable cardioverter-defibrillator (ICD) therapy is considered appropriate when a patient is felt to have a reasonable expectation of 1-year survival. Chronic diseases have been estimated to be associated with greater than $10 \%$ annual mortality and may reduce benefits of ICD therapy. Frailty has been estimated to be associated with greater than $20 \%$ annual mortality and has been suggested to contraindicate ICD therapy.

Purpose: Determine a risk score that may identify patients in whom ICD implantation may not be appropriate.

Methods: Patients who received an ICD for primary and secondary prevention from 2008 through 2013 at the Aurora Health Care network were studied retrospectively. Using Cox regression, a scoring system based on hazard ratios was devised to reflect risk associated with comorbidities. Survival was evaluated by KaplanMeier estimates.

Results: The study cohort includes 1,558 patients (mean age: 61.3 years; 495 female). Comorbidities associated with mortality included in the risk score were need for hemodialysis, myocardial infarct within 3 months prior to ICD implantation, sustained monomorphic ventricular tachycardia, New York Heart Association functional class III, age greater than 70 years, intraventricular conduction delay, diabetes mellitus, and chronic lung disease. A risk score of greater than or equal to 6 was associated with $10 \%$ mortality at 1 year and more than $20 \%$ mortality by 2 years.

Conclusion: Chronic comorbidities have a cumulative effect on mortality. Using our scoring system, patients with a risk score of 6 or greater have at least $10 \%$ mortality at 1 year and more than $20 \%$ mortality by 2 years.

\section{SELECT ABSTRACTS}

Sensitivity of Current Methods for Diagnosing and Documenting Metabolic Syndrome Within a Large Community-Based Health Care System

Tiffany A. Mullen, Danielle M. Greer, Jennifer T. Fink, Renee E. Walker

Department of Integrative Medicine, Aurora Sinai Medical Center; Center for Urban Population Group; Aurora University of Wisconsin Medical Group; Department of Health Informatics and Administration and Zilber School of Public Health, University of Wisconsin-Milwaukee

Background: Metabolic syndrome (MetS) is a constellation of metabolic conditions, including abdominal obesity, high blood pressure, high triglyceride level, low high-density-lipoprotein level and high fasting blood glucose level, that increase the risk of developing chronic health conditions. Various combinations of diagnostic criteria have been proposed, including those by the National Cholesterol Education Program Adult Treatment Panel III (ATP III), World Health Organization (WHO) and International Diabetes Foundation (IDF), among others. However, inconsistent use of diagnostic criteria and inadequate scientific evidence supporting use of specific criteria are current problems in health care.

Purpose: Quantify the prevalence of MetS diagnosis within the Aurora Health Care patient population and determine the sensitivity (ie, accuracy) achieved in documenting MetS within Aurora, recognizing ATP III, WHO and IDF definitions of MetS as diagnostic gold standards.

Methods: We conducted a retrospective review of all patients encountered within Aurora from January 1, 2012, to December 31, 2015. Patients were examined to determine the occurrence and associated dates of MetS diagnosis and all indications of satisfied MetS diagnostic criteria. Indications of obesity, hyperglycemia, hypertriglyceridemia, hypoalphalipoproteinemia and hypertension, as variably defined in ATP III, WHO and IDF guidelines, included relevant diagnoses, abnormal clinical and laboratory test results and use of medications. Sociodemographic data also were collected.

Results: In total, 1,369,620 unique patients visited Aurora during the study period, with $28 \%$ of patients aged $\geq 60$ years and most identifying as non-Hispanic white $(76.8 \%)$ or black $(9.93 \%)$ race. Only 4,978 patients $(0.36 \%)$ received a clinical diagnosis of MetS despite evidence of satisfied ATP III and WHO criteria in $12.0 \%$ and $16.7 \%$ of patients, respectively. Satisfaction of IDF criteria occurred in only $0.16 \%$ of patients. Except for hyperglycemia, individual diagnostic criteria also showed lower-than-expected rates of clinical diagnosis. For example, obesity was diagnosed in $7.86 \%$ of patients but suggested in $39.3 \%$ of patients with body mass index $\geq 30 \mathrm{~kg} / \mathrm{m}^{2}$. The true positive rate (ie, percentage of patients with satisfied criteria who also were clinically diagnosed) was lowest in older, male, black or Hispanic patients. 
Conclusion: Within Aurora, metabolic syndrome is rarely and variably diagnosed in medical practice, despite clear evidence of satisfied ATP III and WHO criteria.

\section{Improved Service Efficiency Improves Racial Disparity in Diabetic Care}

Abel Irena, Kushal Patel, David Thompson, Gregory Schleis, Abiy Gesese, Richard Battiola

\section{Department of Internal Medicine, Aurora Sinai Medical Center; Aurora University of Wisconsin Medical Group}

Background: Racial disparities in diabetes care have been documented. Disparities also have been shown to affect service quality and outcome of diabetic care. Analysis of our internal medicine residency clinic diabetic care management performance across REAL-G (race, ethnicity, age, preferred language and/or gender) showed race-based disparity on two outcome measures: 1) measurement of glycohemoglobin (A1C) at least twice a year; and 2) target blood pressure of $<140 / 90$.

Purpose: Develop interventions to decrease racial disparities in diabetes care among patients managed by an internal medicine residency clinic, as part of the Alliance of Independent Academic Medical Center's National Initiative V project.

Methods: Interventions were developed following analysis of clinic performance data by REAL-G categories, workflow analysis and multidisciplinary clinic team meetings. A point-of-care A1C machine was procured and workflow developed using the Plan-DoStudy-Act cycle. Staff training was conducted. A rolling 12 months data set was obtained from electronic health records. Baseline data range was December 2014 to November 2015, while endline data were from January 2016 to December 2016. The interventions were launched in July 2016. Percentage difference between baseline and endline outcome indicators was calculated and Z-score test assessed. Statistical significance was set at $\mathrm{P}<0.05$.

Results: At baseline, 62.9\% (401 of 638) of patients who selfidentified as African American/black (AA) had A1C measured at least twice a year compared to $74.3 \%$ (107 of 144) of patients who selfidentified as white/Caucasian (WC), a percentage difference of $11.4 \%$ $(\mathrm{P}=0.01)$. For goal blood pressure in diabetics, $71.0 \%$ (453 of 638 ) of AA met the target as compared to $80.6 \%$ (116 of 144) of WC, a percentage difference of $9.6 \%(\mathrm{P}=0.003)$. Following the intervention, a higher percentage of AA patients (71.4\% [381 of 534]) had at least two A1C measured during project period. The outcome also showed improvement for WC (79.8\% [95 of 119]). The percentage difference between races narrowed to $8.5 \%(\mathrm{P}=0.06)$. For goal blood pressure, $75.1 \%$ of AA achieved the target compared to $81.5 \%$ of WC, with percentage difference narrowing to $6.4 \%(\mathrm{P}=0.14)$.

Conclusion: Racial disparities in diabetes were confirmed, even for a clinic setting in which black patients are predominant. Racial disparity can be improved by implementing interventions that improve service for all patients.

\section{Generation of a Patient-Derived Brain Metastasis Breast Cancer Cell Line via Novel Orthotopic Injection Placement and Serial Mouse Transplantation to Develop PDF Mouse Model}

Amber L. LaCrosse, Denise M. Coley, Paul J. Mintz, Santhi D. Konduri, Richard A. Rovin, Amin B. Kassam
Aurora Research Institute; Aurora Neuroscience Innovation Institute

Background: The incidence of brain metastasis appears to be increasing, potentially due to advanced technology that aids early diagnosis. Patient-derived xenografts (PDX) have high translational value, as these models retain key functional characteristics of the patient tumor. PDX models are useful to understand the molecular basis of tumorigenesis and to identify new treatment targets. However, generating a first-line PDX model is challenging as engraftment failure is high. Serial transplanting tumor tissue via mouse-tomouse propagation increases engraftment rates and decreases PDX development time. Herein we report methods to generate a PDX cell line from patient-derived tumor tissue that includes the cerebral aqueduct as a novel intracranial orthotopic implantation site.

Purpose: Develop human tumors in mouse models for therapeutic purpose.

Methods: Patient-derived brain metastasis tumor tissue was enzymatically dissociated into a single cell suspension and maintained in neurocult media supplemented with human recombinant bFGF and EGF $(20 \mathrm{ng} / \mathrm{ml})$. The cells were seeded at a density of $1.0 \times 104 / \mathrm{cm}^{2}$ on ultra-low attachment plates and maintained at $37^{\circ} \mathrm{C}$ with $5 \% \mathrm{CO}_{2}$. PDX models were generated via orthotopic stereotactic surgeries. Athymic nude mice were anesthetized with an intraperitoneal injection of ketamine $(100 \mathrm{mg} / \mathrm{kg})$ and xylazine $(10 \mathrm{mg} / \mathrm{kg})$. The cerebral aqueduct was located using these coordinates from bregma: A: -5 ; L: +0.2 ; V: -2.4 . Mice were injected with $5.0 \times 104$ cells in 2 $\mu \mathrm{l}$ of media at a rate of $0.4 \mathrm{ul} / \mathrm{min}$. Mice were monitored daily for symptoms of tumor formation. Upon becoming symptomatic, mice were euthanized and tumor tissue was harvested for both culture and H\&E stain for tumor verification.

Results: Mice injected with primary patient cells (first-generation mice) developed tumors at 7 weeks (average: 6.77 weeks), secondgeneration mice yielded tumors at 2 weeks (average: 13.5 days), and third-generation mice replicated results from second-generation mice (average: 13 days). H\&E stain revealed invasive tumor masses in the ventricular system that extended from the cerebral aqueduct to the lateral ventricles. Immunohistochemistry analysis confirmed the thirdgeneration cell line retained key characteristics of the patient tumor. Conclusion: These methods successfully generated a PDX cell line from patient-derived brain metastasis that demonstrates reliable tumor formation and phenotypic stability. Importantly, our unique intracranial implantation site revealed several distinct masses, a hallmark of brain metastasis in patients.

\section{Decreasing Time to Broad Spectrum Antibiotics for Septic Patients in the Emergency Department}

Joseph J. Zieminski, Emily E. Bryant

\section{Department of Pharmacy, Aurora St. Luke's Medical Center}

Background: Timely administration of broad spectrum antibiotics has been shown to be directly correlated with decreased mortality for patients with severe sepsis and septic shock. As such, both the Surviving Sepsis Campaign and the SEP-1 CMS measure recognize timely antibiotic administration as a cornerstone of therapy for patients with severe sepsis or septic shock.

Purpose: Decrease time to broad spectrum antibiotic administration for septic patients in the emergency department (ED) of Aurora St. Luke's Medical Center.

Methods: An alert within the electronic medical record was created to more rapidly identify potentially septic patients in the ED. After 